\title{
LANDSCAPE CONNECTIVITY AND SEED DISPERSAL CHARACTERISTICS INFORM THE BEST MANAGEMENT STRATEGY FOR EXOTIC PLANTS
}

Emily S. Minor ${ }^{1,2}$ and Robert H. Gardner ${ }^{1}$

1 Appalachian Laboratory, University of Maryland Center for Environmental Science,

Frostburg, MD 21532, USA

2 Current Address:

University of Illinois at Chicago, 845 W Taylor Street, 3346 SES (M/C 066), Chicago IL 60607

phone: 312-355-0823

e-mail: eminor@uic.edu 


\section{ABSTRACT}

Exotic plant invasions have triggered environmental and economic problems throughout the world. Our ability to manage these invasions is hindered by the difficulty of predicting spread in fragmented landscapes. Because the spatial pattern of invasions depends on the dispersal characteristics of the invasive species and the configuration of suitable habitat within the landscape, a universal management strategy is unlikely to succeed for any particular species. We suggest that the most effective management strategy may be an adaptive one that shifts from local control to landscape management depending on the specific invader and landscape. In particular, we addressed the question of where management activities should be focused to minimize spread of the invading species. By simulating an invasion across a real landscape (Antietam National Battlefield in Maryland, USA), we examined the importance of patch size and connectivity to management success. We found that the best management strategy depended on the dispersal characteristics of the exotic species. Species with a high probability of random long-distance dispersal were best managed by focusing on the largest patches, while species with a lower probability of random long-distance dispersal were best managed by considering landscape configuration and connectivity of the patches. Connectivity metrics from network analysis were useful for identifying the most effective places to focus management efforts. These results provide insight into invasion patterns of various species and suggest a general rule for managers in National Parks and other places where invasive species are a concern.

KEYWORDS: exotic species; invasions; network analysis; graph theory; habitat fragmentation, Antietam National Battlefield 


\section{INTRODUCTION}

Exotic plant invasions have triggered environmental and economic problems throughout the world (Crooks 2002, Ehrenfeld 2003, Duncan et al. 2004). Unfortunately, management of these plants is made more difficult by our limited knowledge of how they spread. Data are especially lacking about the effect of landscape configuration on rate and probability of spread. Nevertheless, we must understand the relationship between pattern and process if we are to anticipate potential invasions and prevent the spread of exotic species into native habitats.

The spatial pattern of invasions depends on the combined characteristics of the invasive species and the configuration of suitable habitat within the landscape. Dispersal may be particularly important, and plants disperse by a variety of means including animal vectors, wind, water, and gravity (Howe and Smallwood 1982). Animals may disperse seeds by ingestion (endozoochorous dispersal), carrying them on their fur, feathers, or feet (epizoochorous dispersal), or by burying them in the ground. While there currently is no consensus in the literature as to which dispersal vector carries propagules farthest, it is quite certain that there is a great deal of variability between species and landscapes (Matlack and Monde 2004, Levey et al. 2008).

Landscape pattern is also likely to be important to invasion outcome. Skellam (1951) used diffusion theory to characterize spread from a single point within a homogeneous landscape. However, real landscapes are not homogeneous and the rate of diffusive spread, which was constant in Skellam's equations, varies depending on the amount and fragmentation of suitable habitat (Bergelson et al. 1993). The dispersal distance of plant propagules is also scale-dependent, with high densities of propagules falling around the parent plant and infrequent, unpredictable long distance dispersal events occurring over larger scales (Moody and Mack 1988). This two-phase dispersal phenomenon, called "infiltration 
invasion" by Wilson and Lee (1989), produces distinct patterns of spread via combinations of short and long distance dispersal. Isolated long-distance dispersal events produce scattered populations that grow with time, increasing the "mass effect" (Shmida and Ellner 1984) of the invasive species and producing and accelerating the rate of invasion. Habitat fragmentation may also affect pattern and rate of invasive spread by creating bottlenecks that funnel invasions into certain areas and/or limit dispersal to more distant habitat areas. Although this general pattern is understood, we currently do not know how to link specific landscape patterns with the unique dispersal characteristics of each species to successfully understand and predict exotic species invasions.

While science cannot yet predict new plant invasions, there are a variety of management options for dealing with existing invasions (Sterling et al. 2004, Flory and Clay 2009). Control methods may include physical removal, chemicals that kill or retard growth, or biological control mechanisms. The location of management actions is also essential and may be selected using spatial or non-spatial criteria. Hobbs and Humphries (1995) suggest selecting locations for management activities based on ecosystem vulnerability and conservation value of the site. While this approach ignores spatial attributes of the invasion process, the authors acknowledge that critical areas may exist where local control can prevent spread to a larger region. An alternative is to reduce the mass effects described by Schmida and Ellner (1984) by focusing control on large populations, regardless of their location, because these populations contribute the greatest number of dispersal propagules to the next generation. This latter approach is often a popular management strategy because large populations are easily detected and may be locally eradicated. Conversely, a spatially explicit strategy that focuses management efforts on satellite populations, rather than single, large, conspicuous infestations (Moody and Mack 1988, Grevstad 2005), is complex and logistically difficult, although Moody and Mack (1988) illustrated that an invader spreads much more rapidly when satellites are ignored. Another spatial strategy is to incorporate 
connectivity, which has been suggested to be an important consideration for management of zebra mussels (Dreissena polymorpha) (Bobeldyk et al. 2009). Finally, Davies and Sheley (2007) discuss methods for incorporating dispersal biology into management strategies, such as limiting plants near vector pathways (e.g., trails, roads, waterways) and minimizing dispersal by specific vectors (e.g., create barriers, clean seeds off clothing and equipment).

The most effective management strategy may be an adaptive one that shifts focus depending on the particular species and the landscape it is invading (Randall 1996). Such an approach must combine knowledge about the dispersal traits of the invader, spatially-explicit data characterizing the invasion, and knowledge of the landscape pattern of suitable habitat. For example, plants with frequent but random long distance dispersal events may spread in unpredictable ways (Higgins and Richardson 1999) and be more difficult to contain; in this case, the best strategy may be to simply focus on managing the largest populations. In contrast, plants with mostly local dispersal may spread in more predictable ways and have easily identifiable bottlenecks where spread may be contained; in that case, the best strategy may be to identify the particular locations on the landscape that may actually slow or stop the spread of the invasion. Modeling is one of the best ways of exploring the impacts of these management strategies on large-scale invasion patterns (Holst et al. 2007, Freckleton et al. 2008). Therefore, we used a simulation model to identify the factors that may cause a shift in management priorities from local control to landscape management. We examine species-specific dispersal attributes and landscape patterns, and address the question of where to focus management activities to maximize overall impact on the invasion.

\section{METHODS}

Study Area \& Map 
The location of this study was Antietam National Battlefield, a 1,317 hectare cultural park in Maryland, U.S.A. Antietam is managed by the National Park Service to resemble the landscape during the U.S. civil war battle of 1862; as a result, the landscape has been preserved as a mosaic of forest fragments, agricultural fields, and pastures. While the landscape was primarily forested prior to European settlement, only about $25 \%$ of the park remains forested today. Exotic species pose a serious problem for natural resource managers in Antietam, where more than 150 nonnative plant species have been found within the park boundaries. In fact, identifying efficient and cost-effective strategies for managing exotic plants is a high priority throughout the National Parks and provides the motivation for this study. Our methods have been developed and tested in Antietam but are intended to be general in nature and useful in a variety of parks and landscapes subject to invasion by nonnative plants.

Map analyses were conducted in ArcGIS 9.2 (ESRI 2006). We identified the forested habitat in Antietam from classified Ikonos satellite imagery ( $4 \mathrm{~m}$ resolution) and subsequently resampled the map to $8 \mathrm{~m}$ resolution to reduce run-time for the simulation model. The map included a $500 \mathrm{~m}$ buffer around park boundaries to incorporate the landscape structure at a broader scale, resulting in a $\sim 4100$ ha map with grid dimensions of 938 rows and 689 columns (Fig. 1). We retained all forested pixels in the map, but only patches greater than 0.5 hectares were selected as 'focal patches' $(\mathrm{n}=42)$ to be targeted for the simulated management efforts described below.

\section{Simulation model}

Exotic plant invasion and management were simulated using CAPS, a stochastic, individualbased, spatially explicit model of plant dispersal and establishment (Plotnick and Gardner 2002, Gardner et al. 2007, Gardner and Engelhardt 2008). In CAPS, each cell in the map may be occupied by a single species with a unique set of life-history traits. The model is discrete in time with a single time step 
representing a yearly cycle of reproduction $(R)$, dispersal $(K)$, and mortality $(d)$ (see Gardner and Engelhardt 2008 for description of other model parameters). A plant may spread to unoccupied cells by two different methods - spatially-constrained neighborhood dispersal governed by dispersal kernels, and low-probability, random long-distance dispersal from occupied cells to any other location within the landscape. Neighborhood dispersal is defined by a species-specific dispersal kernel (e.g., normal, exponential, uniform) with parameters for the mean and maximum dispersal distance; this incorporates frequent local $(\leq$ mean dispersal distance) with infrequent long-distance dispersal events $(\leq$ maximum dispersal distance), as is often seen in empirical dispersal kernels of various species (e.g., Bullock and Clarke 2000, Dovčiak et al. 2008). The probability of random long-distance dispersal is set by the parameter $g$, which when multiplied by the landscape-scale abundance of the invader, $a_{i}$, is the per-cell probability $\left(g a_{i}\right)$ of receiving an invasive species propagule from other occupied cells within the landscape. The parameter $g$ allows us to incorporate the inherent uncertainty in spread patterns associated with long-distance dispersal events (Jones et al. 2005, Clark et al. 2003). Therefore, CAPS includes both a spatially-dependent element of long distance dispersal and a random element of longdistance dispersal.

\section{Focal species}

We simulated a hypothetical pair of woodland species for this analysis. The life-history parameters of these two species were similar: they both had an annual life span $(d=1.0)$, relatively high fecundity $(R=6)$, and an exponential neighborhood dispersal kernel $(K=e)$ with a mean distance of 8 $\mathrm{m}$ (one cell) and a maximum distance of $48 \mathrm{~m}$ (8 cells). To allow us to focus on the importance of random long-distance dispersal events, the two species differed only in the parameter $g$ : Species $\boldsymbol{H}$ had a relatively high probability of random long-distance dispersal $(g=0.001)$ while species $\boldsymbol{L}$ had a 
relatively low, but non-zero, probability $(g=0.0001)$. We simulated annual life spans because there are several common annual exotic species in Antietam forests (e.g., Microstegium vimineum, Veronica persica) and also to speed up computational time; however, our results should not qualitatively differ for species with longer life spans.

We modeled hypothetical species, rather than actual species, for several reasons. First, while limited demographic and dispersal information is available for some exotic plants, the precise estimate of some parameters in CAPS (e.g., R) is essential but cannot be quantitatively estimated with sufficient precision from empirical field measurements (e.g., number of seeds per individual, which may vary greatly among years). Second, we wanted to ensure that our results were general enough to be widely relevant. Therefore, we used realistic, but not species-specific, parameters for this model. The maximum dispersal distance was based on literature suggesting that two habitat patches within 50m of each other would be connected by seed dispersal (Geertsema 2005, Soons et al. 2005), with this distance rounded to $48 \mathrm{~m}$ to accommodate our $8 \mathrm{~m}$ pixel dimensions. Although the actual values of $g$ have not been estimated for any species, the random long-distance dispersal parameters for $H$ and $L$ appear to represent a realistic range of spread rates for invasive plants: higher values $(g>0.001)$ will result in the invading plant dominating the landscape in less than 10 years while lower values $(g<0.0001)$ will require hundreds of years to spread across the park. Many exotic species in the Mid-Atlantic region of the United States have been introduced and subsequently become widespread over this time period (Swearington et al. 2002).

\section{Simulation experiments}

Simulation experiments were performed to evaluate the per patch effectiveness of exotic species management within the Antietam landscape. The management action simulated was the removal of all exotic species at each time step from a single focal patch. For each managed patch, $m_{i}(i=1,42)$, a 
sequential set of invasions were simulated by introducing a single exotic species into one of the remaining 41 patches, $v_{j}(j=1,42 ; j \neq i)$, and allowing it to then spread across the landscape for 50 years (Fig. 1). We performed 10 Monte Carlo iterations for each case, $m_{i} v_{j}$, for a total of $10 \times 42 \times 41=$ 17,220 simulations per species. This sequence of simulations was repeated for both exotic species, $\boldsymbol{H}$ and $\boldsymbol{L}$. Each simulation began on a landscape free of exotic species and with all forested habitat available to be colonized. At the conclusion of each simulation, we recorded the focal patches occupied by the invader and the total amount of habitat (focal patches plus other, smaller areas) occupied as a function of each managed patch.

\section{Analysis with Graph theory}

Graph theory (also called network analysis) is the mathematical study of connectivity or flow in complex networks. Graph theory has been applied in many disciplines and is now recognized as a useful measure of landscape connectivity for ecological studies (Calabrese and Fagan 2004, Minor and Urban 2007, Urban et al. 2009). A graph consists of a set of nodes and connections; in a landscape, nodes are usually defined by habitat patches and connectivity by the movement of organisms between patches. For example, two habitat patches that have seed dispersal between them would be connected while patches beyond the species dispersal range would not be connected. If the dispersal limit of a particular species is known, then a graph can be created to represent landscape connectivity from the perspective of that plant. The topology of that graph then allows identification of the locations on the landscape that most affect patterns of movement - i.e., the hubs or bottlenecks of the graph - and, thus, the locations that may be most suitable for management actions.

Numerous metrics are available for characterizing network structure (Harary 1969, Albert and Barabasi 2002, Urban et al. 2009). Measures of network centrality are useful for our purposes because 
they quantify the structural importance of each node (i.e., habitat patches) within a graph (Borgatti 2005, Crucitti et al. 2006). We used three measures of centrality to describe each focal patch: degree, betweenness, and closeness. Degree centrality (D) is the number of connections for a given habitat patch. Betweenness centrality (B) is an indicator of how frequently movement may occur through a particular habitat patch, defined as the number of shortest paths that pass through the node of interest. Finally, closeness centrality $(C)$ is a measure of how close a habitat patch is to all other patches in the landscape. $\mathrm{C}$ is defined as the mean shortest path distance from a given node to all other nodes in the graph. D measures connectivity at a local scale, while B and C are patch attributes measuring connectivity across the entire landscape.

We analyzed the structure of the Antietam landscape and, using the 48m maximum seed dispersal distance from the dispersal kernel, constructed a landscape graph (Fig 2). This graph assumes that forest patches within $48 \mathrm{~m}$ of each other (their edge-to-edge distance) are connected regardless of the intervening habitat separating these patches. The centrality measures $(\mathrm{D}, \mathrm{B}, \mathrm{C})$ for each focal habitat patch were then calculated using Pajek 1.20 software (Batagelj and Mrvar 1996). Finally, the three graph metrics were used as predictor variables of management effectiveness in the regression model created to summarize simulation results (described below).

\section{Analysis of simulation results}

Data produced by the 17,220 simulations were analyzed to determine the relative effectiveness of patch-specific management actions for slowing or preventing the spread of invasive species. We also wanted to assess the utility of pattern measures based on graph theory to predict management outcome, eliminating the necessity of extensive model simulations. To accomplish these objectives, we used a linear regression model to statistically relate the total abundance of invading species (A) after a 50 year 
invasion period with four metrics describing each managed patch, $m_{i}$ : the size of the patch (S) and its degree (D), betweenness (B), and closeness (C). The regression model was iteratively applied based on the ease of measuring each predictor variable: $\mathrm{S}$ is simple to calculate either directly or via mapping software (i.e., GIS); D is also easily calculated by GIS analysis as simple counts of patches that fall within a requisite buffer distance; $\mathrm{C}$ and $\mathrm{B}$ require a bit more expertise and the use of graph-analysis software such as Pajek (Batagelj and Mrvar 1996). Therefore, we tested a sequence of nested hypotheses from simple $(\mathrm{A}=f(\mathrm{~S}))$ to complex $(\mathrm{A}=f(\mathrm{~S}+\mathrm{D}+\mathrm{C}+\mathrm{B}))$ to identify the best linear regression model for predicting management outcome. Predictor variables were always added in order of measurement ease, and the regression sum of squares was tested with each added predictor variable to verify that the new term significantly improved the predictive power of the model. New predictor variables were only included if the null hypothesis - that the added predictor variable did not increase explanatory power of the model — was rejected. This allowed us to identify the most straightforward and simple model that would also have high utility to managers. Statistical analysis was performed with R (R Development Core Team. 2008) using the general linear model procedure.

\section{RESULTS}

In our simulations, three variables governed the rate and extent of dispersal - the probability of random long-distance dispersal $(g)$, the initial introduction point of the exotic species, $v_{j}$, and the location of exotic plant management, $m_{j}$. The importance of random long-distance dispersal events to invasion outcome was clear. Species $\boldsymbol{H}$, with high probability of random long-distance dispersal, spread much farther on average than did species $\boldsymbol{L}$. After the 50-year simulations, a mean of $12 \%$ (range 0.1 $49 \%$ ) of the forested habitat was invaded by species $\boldsymbol{L}$ while a mean of $47 \%$ (range $3-76 \%$ ) of the habitat was invaded by species $\boldsymbol{H}$ (Fig. 3). The distribution of the response variable (abundance across 
the landscape) was right-skewed for species $\boldsymbol{L}$, with most simulations resulting in very little habitat occupied while a few simulations resulted in over $40 \%$ of the habitat being invaded. Abundance was more normally distributed for species $\boldsymbol{L}$ and the range of output values was larger, indicating greater variability in the extent of invasion when random long-distance dispersal was more common. The variability in model output reflects stochastic model processes as well as the unique dynamics that occur when management and invasion happen in diverse locations across the landscape.

The initial introduction point of the exotic species, $v_{j}$, was also very important to the rate and extent of invasion. Some introduction locations resulted in much more extensive invasions than other locations. For simulations with species $\boldsymbol{L}$, the mean abundance ranged from $1 \%$ of the landscape, when the invasion was initiated in a small or poorly connected patch, to $53 \%$ of the landscape when the invasion was initiated in a large or well-connected patch. For simulations with species $\boldsymbol{H}$, the mean abundance for each introduction point ranged from $15 \%$ to $84 \%$ of the landscape. To eliminate bias from introduction location in our analysis of management strategies, we averaged model output over all introduction locations in subsequent analyses.

Finally, the choice of management location, $m_{i}$, had a profound effect on the rate and extent of invasion across the landscape. When averaged over all introduction points, management in some locations resulted in less extensive invasions than management in other locations. For simulations with species $\boldsymbol{L}$, the mean abundance ranged from $8 \%$ of the landscape, when management was carried out in a large or well-connected patch, to $14 \%$ when management occurred in a small or poorly-connected patch. For simulations with species $\boldsymbol{H}$, the mean abundance ranged from $27 \%$ to $57 \%$ of the landscape.

Graph metrics were all highly correlated with each other and with patch size (Table 1; analyses done in R). Mean degree centrality over all focal patches was 13 (range 0-99), mean betweenness was 
0.01 (range 0-0.11), and mean closeness was 0.03 (range 0-0.09). Model output (invader abundance after 50 years) was significantly correlated with each metric. For simulations with species $\boldsymbol{H}$, model output was most correlated with size of the managed patch $(\mathrm{r}=-0.98)$. For simulations with species $\boldsymbol{L}$, model output was most correlated with degree of the managed patch $(r=-0.94)$.

Management in larger or more connected patches slowed the invasion more than management in smaller or less connected patches. Management outcome for species $\boldsymbol{L}$ was best predicted by the full regression model with all predictor variables, which explained $93 \%$ of the variability in the response variable (Table 2). Each predictor variable, when added to the model sequentially, made a significant contribution to the model, although the majority of the predictive power came from the first two variables — patch size and degree. In fact, we could attain a very high level of predictive ability with a simple linear regression using degree (D) as the only predictor variable $\left(r^{2}=0.88\right)$. Management outcome for species $\boldsymbol{H}$ was best predicted with only two variables_-patch size and degree $\left(\mathrm{r}^{2}=0.97\right.$; Table 3$)$. However, a simple regression model with only patch size $(\mathrm{S})$ as a predictor was very similar in predictive ability $\left(\mathrm{r}^{2}=0.96\right)$.

A closer look at some individual model runs illustrates how potential management activities can slow the spread of an invader that has a low probability of random long-distance dispersal. Figure 4 shows a model run with management for species $\boldsymbol{L}$ occurring in the habitat patch with the highest degree centrality_patch 41 . When the invasion was initiated in patches either north or south of patch 41 , the invader did not spread beyond this managed patch after 50 years: patch 41 acted as a bottleneck for the invasion, and when vigilant management activities occurred at that location (i.e., the exotic species was completely eradicated), spread was limited to the side of the park where that invasion originated. In contrast, Figure 5 shows the same management strategy with species $\boldsymbol{H}$. When random long-distance 
dispersal events were frequent (as indicated by dispersal parameters for species $\boldsymbol{H}$ ), patch 41 no longer acted as a bottleneck for the invasion. Not only did the invader spread more quickly, but the pattern of spread became less predictable.

\section{DISCUSSION}

Our results provide new insight into invasion patterns of exotic plants and suggest a general rule for managers wishing to control the spread and extent of habitat occupied by invasive species. We have shown that when an invading plant frequently and randomly disperses its seeds over long distances, spread is not constrained by landscape connectivity. In those cases, the best management strategy is to focus on the largest patches — which would become the largest populations if unmanaged —in order to keep overall abundance as low as possible. However, if an invader fails to demonstrate a tendency toward random long-distance dispersal events, the best way to slow or prevent spread of that species is to monitor and manage habitat patches that are large but also highly connected to the rest of the landscape. In other words, a non-spatial strategy appears to be best for species with frequent random long-distance dispersal events but a spatially-explicit strategy may be best for species with mostly local dispersal.

Introduced species often cannot be eradicated from established locations. In these cases, the next-best approach is to stop or slow the spread into new areas. Using the jargon of network theory, spread can sometimes be limited by identifying bottlenecks, which are locations on the landscape that constrict movement, or hubs, which are highly-connected patches. We successfully used network metrics to identify these important connection points on the landscape and reduce the extent of the invasion. This approach is similar to the "barrier zone" method applied by Sharov and Liebhold (1998) to slowing the spread of gypsy moth (Lymantria dispar). Our results indicate that, as long as the 
possibility of random long-distance dispersal exists, it is not realistic to completely prevent spread of a species in this way. Furthermore, when probability of random long-distance dispersal is high, this approach is less successful than simply targeting the largest patches. However, for species with infrequent random long-distance dispersal, this method can delay colonization of uninvaded areas for an uncertain period of time. While continual management in hubs may be key to preventing spread of species with low probabilities of random long-distance dispersal, given two equally-connected patches of different size, the obvious choice is to focus immediate management efforts on the larger patch to reduce overall abundance of the exotic species across the landscape.

These strategies may prove useful for the National Parks, which spend large amounts of money and time managing exotic plants. The National Parks System has 16 regional Exotic Plant Management Teams (EPMTs) that assist the parks in preventing introductions of new species, reducing existing infestations, and restoring native plant communities. In Antietam, as in other parks in the region, the EPMT does not manage the entire park but instead has discrete management units where they repeatedly return to monitor and treat exotic plant populations. Therefore, our simulations of persistent management in one location are not unrealistic. In fact, our results validate this approach if the focal species has a low probability of random long-distance dispersal and the management unit is a highlyconnected hub in the landscape.

Few studies have examined the effect of dispersal characteristics on management success. While our findings have great implications for management decisions, they beg the question "Which species have frequent random long-distance dispersal events?" This question is not easy to answer, since long distance dispersal events are rarely recorded. However, a few landscape and species characteristics may be helpful for determining how often a seed travels far beyond the parent plant. First, animal dispersed 
seeds may be more likely to travel farther than seeds that are dispersed by abiotic means (Clark et al. 2005, Vittoz and Engler 2007, Minor et al. 2009). Animals behave in complex ways that may result in apparently-random long distance dispersal events of seeds inside their gut or attached externally to fur or feathers (Russo et al. 2006). In particular, volant animals (birds and bats) and large mammals (e.g., deer) are likely to carry seeds longer distances than smaller terrestrial animals such as rodents. Second, any plant that is dispersed by humans is more likely to be carried far away from the parent plant (Vittoz and Engler 2007, Wichmann et al. 2009). Dispersal by humans may be intentional, such as ornamental plants sold in plant nurseries, or it may be unintentional. Unintentional dispersal can occur when a seed becomes embedded in the mud in a hiker's shoe or a car or bicycle tire, and is much more likely to happen to plants with small seeds than plants with large seeds. Therefore, plants with large seeds (e.g., acorns) or those that are dispersed by gravity, water, or wind may be less likely to experience random long-distance seed dispersal events than their counterparts.

While we lack historical information about invasions, we can use empirical data from a previous study (Minor et al. 2009) to examine the dispersal mechanisms of the most and least widespread exotic plants in Antietam upland forests. Of the five exotic species that were most widespread, four are dispersed by animal ingestion (Lonicera japonica, Rosa multiflora, Duchesnea indica, Rubus phoenicolasius) and one is cultivated for food and medicinal use (i.e., dispersed by humans: Alliara petiolata). Conversely, of the 27 species that only occurred in a single sample location (i.e., the least widespread exotic species), four were dispersed by adhesion, two were ant-dispersed, two were dispersed by ingestion, six were wind-dispersed, and 13 had no obvious dispersal mechanism. In other words, widespread species tended to be animal- or human-dispersed, while species with limited distribution tended to be spread by abiotic means. Unfortunately, we lack detailed information about the rate and spatial pattern of spread for each of these species and thus cannot directly test our ideas about 
how dispersal mechanism affects spread. However, lacking this information, we can infer that species which are currently widespread in Antietam may have spread more rapidly in the past and been less constrained by connectivity in the fragmented landscape. Testing these assumptions would be a fruitful direction for future research.

We also speculate that the probability of random long-distance dispersal depends upon the composition and configuration of the invaded landscape. This may be especially important for animaldispersed seeds, as it has been shown that animal foraging, dispersal, and movement patterns depend on landscape configuration (Etzenhouser et al. 1998, Selonen et al. 2001, Farwig et al. 2006, Levey et al. 2008). However, many species with other presumed dispersal modes (i.e., wind dispersal) are also dispersed by animals (Myers et al. 2004), and consequently dispersal distance of those species will change as animal movement patterns change. It has also been shown that dispersal distance of an animal-dispersed species may depend upon density of conspecific plants (Carlo and Morales 2008) or the animal community in the surrounding landscape (Bleher and Bohning-Gaese 2001). Long-distance dispersal by abiotic means is likely to be landscape-specific as well. For example, long-distance dispersal by water is more likely to occur in landscapes that are prone to flooding (Tabacchi et al. 2005, Nakayama et al. 2007). For wind-dispersed species, regions with higher wind speed or more frequent storms or updrafts may have more long-distance dispersal events (Bullock and Clarke 2000, Tackenberg et al. 2003); in turn, wind speeds are affected by vegetation patterns on the landscape (Nuttle and Haefner 2005). Finally, species that are dispersed by humans will likely have a greater probability of random long-distance dispersal in landscapes with more intense human disturbance. Comparing spread rates of a single species through different landscapes may be helpful for testing some of these ideas. 
Seed dispersal is not the only thing that should affect management strategy. The timing of the invasion is also important to consider. For example, Hobbs and Humphries (1995) suggested three different strategies depending upon stage of invasion. For the newest introductions, they suggest that quarantine may be the most appropriate tactic. When an invasion has progressed to multiple populations and nascent foci, they recommend preventing spread by early control and eradication. Finally, in the late stage of invasion, when the exotic species has already spread across the landscape, they suggest an integrated ecosystem management approach. This paper provides some guidance on how to select management locations for species in the intermediate stages of an invasion. Once these locations have been identified, the appropriate management technique (e.g., spraying, cutting, etc.) would depend on the focal species (DiTomaso 2000, Myers et al. 2000). Other approaches, such as those described by Hobbs and Humphries (1995) or Theoharides and Dukes (2007) would be more appropriate at different stages of the invasion.

The nature of ecological models is to simplify complex processes and our simulations are no exception. There are many factors that may limit the spread of an exotic species in addition to the frequency of random long-distance dispersal events. For example, unsuitable environmental conditions or competition from native plant communities may prevent establishment of exotic plants (Brothers and Spingarn 1992, D'Antonio 1993, Bartuszevige et al. 2007, Theoharides and Dukes 2007). Propagule pressure is also clearly important to the success of exotic species invasions (Holle and Simberloff 2005, Lockwood et al. 2005, Colautti et al. 2007). In addition, we found that the location of an introduction event had a large impact on the spread of the invader in our model. It is not surprising that other studies have found similar results (Ferrari and Lookingbill 2009), as an exotic species introduced into an isolated or unsuitable habitat patch is unlikely to spread beyond the initial location. In the Antietam landscape, every habitat patch was considered suitable for establishment. While this may or may not 
reflect actual conditions, holding habitat suitability constant allowed us to examine the spatial patterns that might otherwise be overwhelmed by local variability in environmental attributes.

In conclusion, there is a real need to provide practical, simple, and science-based tools to managers of natural areas (D'Antonio et al. 2004). Our goal was to provide such a tool, and we found that different management strategies may be appropriate for different exotic plant species and different landscapes. If the invader is known or suspected to have a high probability of random long-distance dispersal, management efforts may be best focused on the largest patches. With these species, preventing further spread is difficult and the most successful approach may be to simply minimize the population size across the landscape. Conversely, if the invader has a low probability of random longdistance dispersal, spread can be slowed or stopped by incorporating simple measures of landscape connectivity into the management plan. For the latter species, spatial management strategies can be very useful. These hypotheses need to tested and verified with field surveys but, if supported with empirical data, will provide a convenient rule-of-thumb for managers and new insight into invasion patterns of exotic plants.

\section{ACKNOWLEDGEMENTS}

We thank scientists and staff at the Center for Urban Ecology for their support and suggestions throughout this project. Two anonymous reviewers provided valuable feedback on an earlier version of the manuscript. Funding was provided to ESM by the National Parks Ecological Research program. 


\section{REFERENCES}

Albert, R., and A. L. Barabasi. 2002. Statistical mechanics of complex networks. Reviews Of Modern Physics 74:47-97.

Bartuszevige, A. M., R. L. Hrenko, and D. L. Gorchov. 2007. Effects of leaf litter on establishment, growth and survival of invasive plant seedlings in a deciduous forest. American Midland Naturalist 158:472-477.

Batagelj, V., and A. Mrvar. 1996. Pajek 1.20.

Bergelson, J., J. A. Newman, and E. M. Floresroux. 1993. Rate of weed spread in spatially heterogeneous environments. Ecology 74:999-1011.

Bleher, B., and K. Bohning-Gaese. 2001. Consequences of frugivore diversity for seed dispersal, seedling establishment and the spatial pattern of seedlings and trees. Oecologia 129:385394.

Bobeldyk, A. M., J. M. Bossenbroek, M. A. Evans-White, D. M. Lodge, and G. A. Lamberti. 2009. Secondary spread of zebra mussels (Dreissena polymorpha) in coupled lake-stream systems. Ecoscience 12:339-346.

Borgatti, S. P. 2005. Centrality and network flow. Social Networks 27:55-71.

Brothers, T. S., and A. Spingarn. 1992. Forest Fragmentation and Alien Plant Invasion of Central Indiana Old-Growth Forests. Conservation Biology 6:91-100.

Bullock, J. M., and R. T. Clarke. 2000. Long distance seed dispersal by wind: measuring and modelling the tail of the curve. Oecologia 124:506-521.

Calabrese, J. M., and W. F. Fagan. 2004. A comparison-shopper's guide to connectivity metrics. Frontiers in Ecology and the Environment 2:529-536. 
Carlo, T. A., and J. M. Morales. 2008. Inequalities in fruit-removal and seed dispersal: consequences of bird behaviour, neighbourhood density and landscape aggregation. Journal of Ecology 96:609-618.

Clark, C. J., J. R. Poulsen, B. M. Bolker, E. F. Connor, and V. T. Parker. 2005. Comparative seed shadows of bird-, monkey-, and wind-dispersed trees. Ecology 86:2684-2694.

Clark, J.S., M. Lewis, J.S. McLachlan, and J. HilleRisLambers. 2003. Estimating population spread: what can we forecast and how well? Ecology 84: 1979-1988.

Colautti, R. I., I. A. Grigorovich, and H. J. MacIsaac. 2007. Propagule pressure: a null model for biological invasions. Biological Invasions 9:885-885.

Crooks, J. A. 2002. Characterizing ecosystem-level consequences of biological invasions: the role of ecosystem engineers. Oikos 97:153-166.

Crucitti, P., V. Latora, and S. Porta. 2006. Centrality measures in spatial networks of urban streets. Physical Review E 73.

D'Antonio, C. M. 1993. Mechanisms Controlling Invasion of Coastal Plant Communities by the Alien Succulent Carpobrotus Edulis. Ecology 74:83-95.

D'Antonio, C. M., N. E. Jackson, C. C. Horvitz, and R. Hedberg. 2004. Invasive plants in wildland ecosystems: merging the study of invasion processes with management needs. Frontiers in Ecology and the Environment 2:513-521.

Davies, K. W., and R. L. Sheley. 2007. A conceptual framework for preventing the spatial dispersal of invasive plants. Weed Science 55:178-184.

DiTomaso, J. M. 2000. Invasive weeds in rangelands: Species, impacts, and management. Weed Science 48:255-265. 
Dovčiak, M., R. Hrivnák, K. Ujházy, and D. Gömöry. 2008. Seed rain and environmental controls on invasion of Picea abies into grassland. Plant Ecology 194: 135-148.

Duncan, C. A., J. J. Jachetta, M. L. Brown, V. F. Carrithers, J. K. Clark, J. M. Ditomaso, R. G. Lym, K. C. McDaniel, M. J. Renz, and P. M. Rice. 2004. Assessing the economic, environmental, and societal losses from invasive plants on rangeland and wildlands. Weed Technology 18:1411-1416.

Ehrenfeld, J. G. 2003. Effects of exotic plant invasions on soil nutrient cycling processes. Ecosystems 6:503-523.

Etzenhouser, M. J., M. K. Owens, D. E. Spalinger, and S. B. Murden. 1998. Foraging behavior of browsing ruminants in a heterogeneous landscape. Landscape Ecology 13:55-64.

Farwig, N., K. Bohning-Gaese, and B. Bleher. 2006. Enhanced seed dispersal of Prunus africana in fragmented and disturbed forests? Oecologia 147:238-252.

Ferrari, J. R., and T. R. Lookingbill. 2009. Initial conditions and their effect on invasion velocity across heterogeneous landscapes. Biological Invasions 11:1247-1258.

Flory, S. L., and K. Clay. 2009. Invasive plant removal method determines native plant community responses. Journal of Applied Ecology 46:434-442.

Freckleton, R. P., W. J. Sutherland, A. R. Watkinson, and P. A. Stephens. 2008. Modelling the effects of management on population dynamics: some lessons from annual weeds. Journal of Applied Ecology 45:1050-1058.

Gardner, R. H., and K. A. M. Engelhardt. 2008. Spatial processes that maintain biodiversity in plant communities. Perspectives in Plant Ecology, Evolution and Systematics 9:211-228. 
Gardner, R. H., J. D. Forester, and R. E. Plotnick. 2007. Determining pattern-process relationships in heterogeneous landscapes. Pages 92-114 in J. Wu and R. Hobbs, editors. Key Topics and Perspectives in Landscape Ecology. Cambridge University Press.

Geertsema, W. 2005. Spatial dynamics of plant species in an agricultural landscape in the Netherlands. Plant Ecology 178:237-247.

Grevstad, F. S. 2005. Simulating control strategies for a spatially structured weed invasion: Spartina alterniflora (Loisel) in Pacific Coast estuaries. Biological Invasions 7:665-677.

Harary, F. 1969. Graph Theory. Addison-Wesley, Massachusetts.

Higgins, S. I., and D. M. Richardson. 1999. Predicting Plant Migration Rates in a Changing World: The Role of Long-Distance Dispersal. The American Naturalist 153:464-475.

Hobbs, R. J., and S. E. Humphries. 1995. An Integrated Approach to the Ecology and Management of Plant Invasions. Conservation Biology 9:761-770.

Holle, B. V., and D. Simberloff. 2005. ECOLOGICAL RESISTANCE TO BIOLOGICAL INVASION OVERWHELMED BY PROPAGULE PRESSURE. Ecology 86:3212-3218.

Holst, N., I. A. Rasmussen, and L. Bastiaans. 2007. Field weed population dynamics: a review of model approaches and applications. Weed Research 47:1-14.

Howe, H. F., and J. Smallwood. 1982. Ecology Of Seed Dispersal. Annual Review of Ecology and Systematics 13:201-228.

Jones, F.A., J. Chen, G.-J. Weng, and S.P. Hubbell. 2005. A genetic evaluation of seed dispersal in the Neotropical tree Jacaranda copaia (Bignoniaceae). The American Naturalist 166: 543-55.

Levey, D. J., J. J. Tewksbury, and B. M. Bolker. 2008. Modelling long-distance seed dispersal in heterogeneous landscapes. Journal of Ecology 96:599-608. 
Lockwood, J. L., P. Cassey, and T. Blackburn. 2005. The role of propagule pressure in explaining species invasions. Trends in Ecology \& Evolution 20:223-228.

Matlack, G. R., and J. Monde. 2004. Consequences of low mobility in spatially and temporally heterogeneous ecosystems. Journal of Ecology 92:1025-1035.

Minor, E. S., and D. Urban. 2007. Graph theory as a proxy for spatially explicit population models in conservation planning. Ecological Applications 17:1771-1782.

Minor, E. S., S. M. Tessel, K. A. M. Engelhardt, and T. R. Lookingbill. 2009. The role of landscape connectivity in assembling exotic plant communities: a network analysis. Ecology 90:1802-1809.

Moody, M. E., and R. N. Mack. 1988. Controlling the Spread of Plant Invasions: The Importance of Nascent Foci. Journal of Applied Ecology 25:1009-1021.

Myers, J. A., M. Vellend, S. Gardescu, and P. L. Marks. 2004. Seed dispersal by white-tailed deer: implications for long-distance dispersal, invasion, and migration of plants in eastern North America. Oecologia 139:35-44.

Myers, J. H., D. Simberloff, A. M. Kuris, and J. R. Carey. 2000. Eradication revisited: dealing with exotic species. Trends in Ecology \& Evolution 15:316-320.

Nakayama, N., J. Nishihiro, Y. Kayaba, T. Muranaka, and I. Washitani. 2007. Seed deposition of Eragrostis curvula, an invasive alien plant on a river floodplain. Ecological Research 22:696-701.

Nuttle, T., and J. W. Haefner. 2005. Seed dispersal in heterogeneous environments: Bridging the gap between mechanistic dispersal and forest dynamics models. American Naturalist 165:336-349. 
Plotnick, R. E., and R. H. Gardner. 2002. A general model for simulating the effects of landscape heterogeneity and disturbance on community patterns. Ecological Modelling 147:171197.

R Development Core Team. 2008. R: A language and environment for statistical computing. R Development Core Team. R Foundation for Statistical Computing, Vienna, Austria.

Randall, J. M. 1996. Weed control for the preservation of biological diversity. Weed Technology 10:370-383.

Russo, S. E., S. Portnoy, and C. K. Augspurger. 2006. Incorporating animal behavior into seed dispersal models: Implications for seed shadows. Ecology 87:3160-3174.

Selonen, V., I. K. Hanski, and P. C. Stevens. 2001. Space use of the Siberian flying squirrel Pteromys volans in fragmented forest landscapes. Ecography 24:588-600.

Sharov, A. A., and A. M. Liebhold. 1998. Model of slowing the spread of gypsy moth (Lepidoptera : Lymantriidae) with a barrier zone. Ecological Applications 8:1170-1179.

Shmida, A., and S. Ellner. 1984. Coexistence of Plant-Species with Similar Niches. Vegetatio 58:29-55.

Skellam, J. B. 1951. Random dispersal in theoretical populations. Biometrika 38:196-218.

Soons, M. B., J. H. Messelink, E. Jongejans, and G. W. Heil. 2005. Habitat fragmentation reduces grassland connectivity for both short-distance and long-distance wind-dispersed forbs. Journal of Ecology 93:1214-1225.

Sterling, T. M., D. C. Thompson, and L. B. Abbott. 2004. Implications of invasive plant variation for weed management. Weed Technology 18:1319-1324. 
Swearingen, J., K. Reshetiloff, B. Slattery, and S. Zwicker. 2002. Plant Invaders of Mid-Atlantic Natural Areas. National Park Service and U.S. Fish \& Wildlife Service, Washington, D.C. 82 pp.

Tabacchi, E., A. M. Planty-Tabacchi, L. Roques, and E. Nadal. 2005. Seed inputs in riparian zones: Implications for plant invasion. Pages 299-313. John Wiley \& Sons Ltd.

Tackenberg, O., P. Poschlod, and S. Kahmen. 2003. Dandelion seed dispersal: The horizontal wind speed does not matter for long-distance dispersal - it is updraft! Plant Biology 5:451-454.

Theoharides, K. A., and J. S. Dukes. 2007. Plant invasion across space and time: factors affecting nonindigenous species success during four stages of invasion. New Phytologist 176:256-273.

Urban, D. L., E. S. Minor, E. A. Treml, and R. S. Schick. 2009. Graph models of habitat mosaics. Ecology Letters 12:260-273.

Vittoz, P., and R. Engler. 2007. Seed dispersal distances: a typology based on dispersal modes and plant traits. Botanica Helvetica 117:109-124.

Wichmann, M. C., M. J. Alexander, M. B. Soons, S. Galsworthy, L. Dunne, R. Gould, C. Fairfax, M. Niggemann, R. S. Hails, and J. M. Bullock. 2009. Human-mediated dispersal of seeds over long distances. Proceedings of the Royal Society B-Biological Sciences 276:523-532.

Wilson, J. B., and W. G. Lee. 1989. Infiltration Invasion. Functional Ecology 3:379-380. 


\section{TABLES}

Table 1. Correlation coefficients for patch metrics and CAPS output (i.e., abundance). Patch metrics describe the patch where management was performed, $m_{i}$. Abundance indicates the extent of the invasion after 50 simulation years with high (Species $\boldsymbol{H}$ ) or low (Species $\boldsymbol{L}$ ) probability of random longdistance seed dispersal. All values are statistically significant at $\mathrm{p}=0.01$.

\begin{tabular}{|c|c|c|c|c|c|}
\hline & Degree & Closeness & Betweenness & Abundance $_{\text {Species } L}$ & Abundance $_{\text {Species } H}$ \\
\hline Patch Size & 0.87 & 0.43 & 0.64 & -0.88 & -0.98 \\
\hline Patch Degree & & 0.58 & 0.87 & -0.94 & -0.89 \\
\hline Patch Closeness & & & 0.68 & -0.66 & -0.49 \\
\hline Patch Betweenness & & & & -0.85 & -0.68 \\
\hline Abundance $_{\text {Species } L}$ & & & & & 0.92 \\
\hline
\end{tabular}


Table 2. Sequential analysis of variance for linear regression model predicting management success (abundance of exotic species after 50 simulation years) for species with low probability of long distance dispersal (Species $\boldsymbol{L}$ ). The best model is one that includes patch size and the connectivity metrics.

\begin{tabular}{lcllll}
\hline Model terms added sequentially & d.f. & Sum of Sq. & F Value & p $(\mathbf{F})$ & $\mathbf{r}^{2}$ \\
\hline Patch Size* & 1 & 0.64 & 428.9 & $<<0.0001$ & 0.78 \\
Patch Degree* & 1 & 0.10 & 67.4 & $<<0.0001$ & 0.90 \\
Patch Closeness* & 1 & 0.02 & 14.9 & 0.0004 & 0.92 \\
Patch Betweenness* & 1 & 0.01 & 4.9 & 0.03 & 0.93 \\
\hline
\end{tabular}

* Metrics describe the patch where management was performed, $m_{i}$ 
Table 3. Sequential analysis of variance for linear regression model predicting management success (abundance of exotic species after 50 simulation years) for species with high probability of long distance dispersal (Species $\boldsymbol{H}$ ). The best model includes only patch size and degree, and degree adds minimal explanatory power.

\begin{tabular}{lcllll}
\hline Model terms added sequentially & d.f. & Sum of Sq. & F Value & p (F) & $\mathbf{r}^{\mathbf{2}}$ \\
\hline Patch Size* & 1 & 17.91 & 1130.4 & $<<0.0001$ & 0.96 \\
Patch Degree* & 1 & 0.13 & 8.5 & 0.006 & 0.97 \\
Patch Closeness* & 1 & 0.02 & 1.5 & $\mathrm{~ns}$ & 0.97 \\
Patch Betweenness* & 1 & 0.01 & 0.5 & $\mathrm{~ns}$ & 0.97 \\
\hline
\end{tabular}

* Metrics describe the patch where management was performed, $m_{i}$ 


\section{FIGURE LEGENDS}

Figure 1. Time series of simulated invasion in Antietam National Battlefield (Maryland, U.S.A.). Light gray shaded area is park boundary; darker gray areas are forest habitat; black areas have been invaded by the exotic species.

Figure 2. Network of forest patches in and around Antietam National Battlefield. Light gray area shows park boundaries; darker gray areas are forested. Forest patches are connected to each other by lines if they are within $48 \mathrm{~m}$ of each other, indicating connectivity by seed dispersal.

Figure 3. Histogram of simulation model output. The top panel shows the simulation output for species $\boldsymbol{L}$, which has lower levels of random long-distance seed dispersal. The bottom panel shows simulation output for species $\boldsymbol{H}$, which has higher levels of random long-distance seed dispersal.

Figure 4. Output from two model runs with species $\boldsymbol{L}$ where management occurred in the patch with the highest degree centrality. The arrow shows the habitat patch where the invasion began; the striped habitat is where management activities took place (patch 41). Dark patches have been invaded after 50 years; light patches are uninvaded. In both panels (invasion initiated either north or south of patch 41) management in patch 41 prevented spread to the other side of the park. Contrast to Figure 5.

Figure 5. Output from two model runs with species $\boldsymbol{H}$ where management occurred in the patch with the highest degree centrality. The arrow shows the habitat patch where the invasion began; the striped habitat is where management activities took place (patch 41). Dark patches have been invaded after 50 years; light patches are uninvaded. In contrast to Fig. 4, management in patch 41 does not prevent spread 
of the invader from one side of the park to the other when the invader experiences frequent long distance dispersal events. 
FIGURES
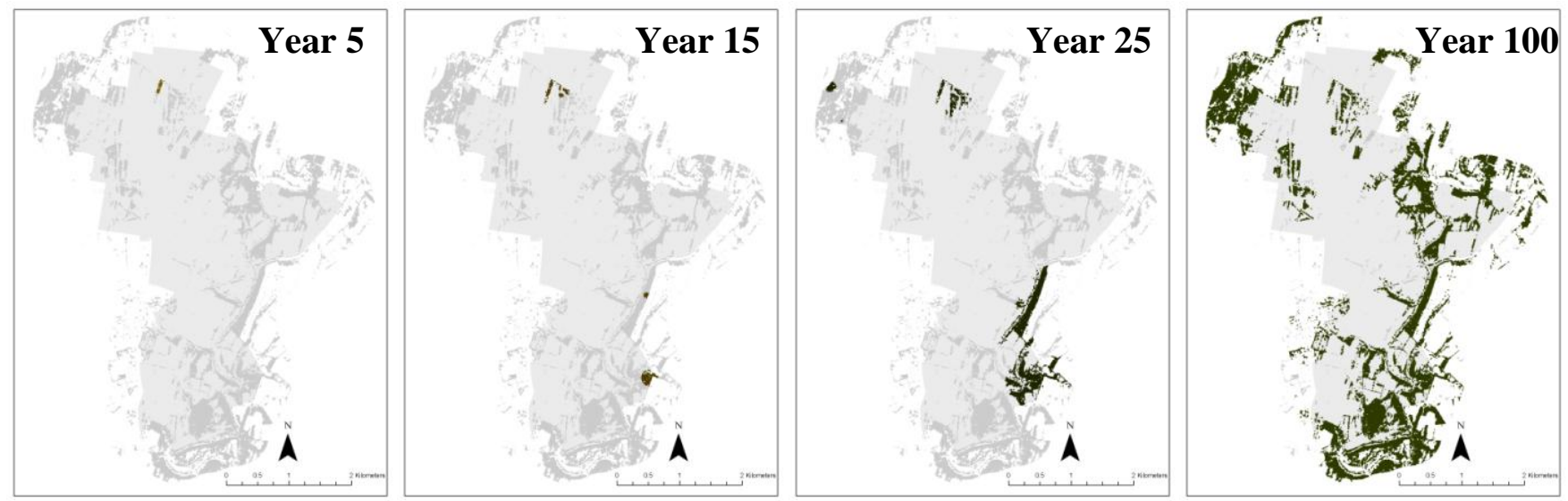

Figure 1 


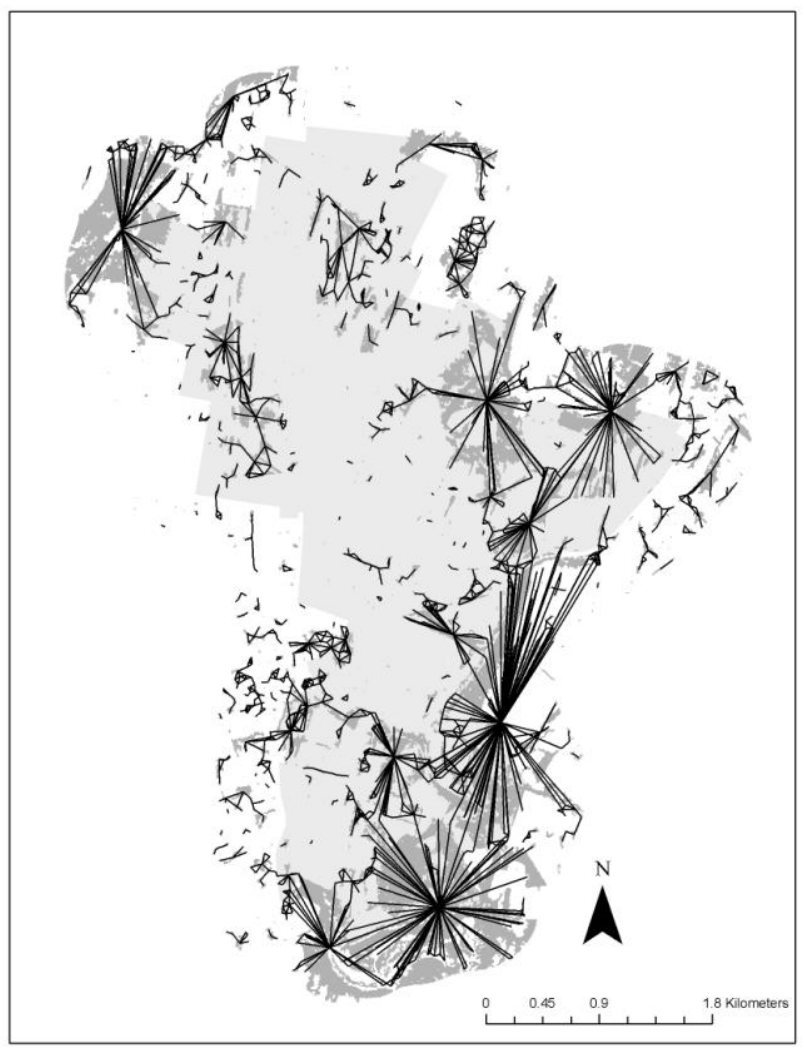

Figure 2 

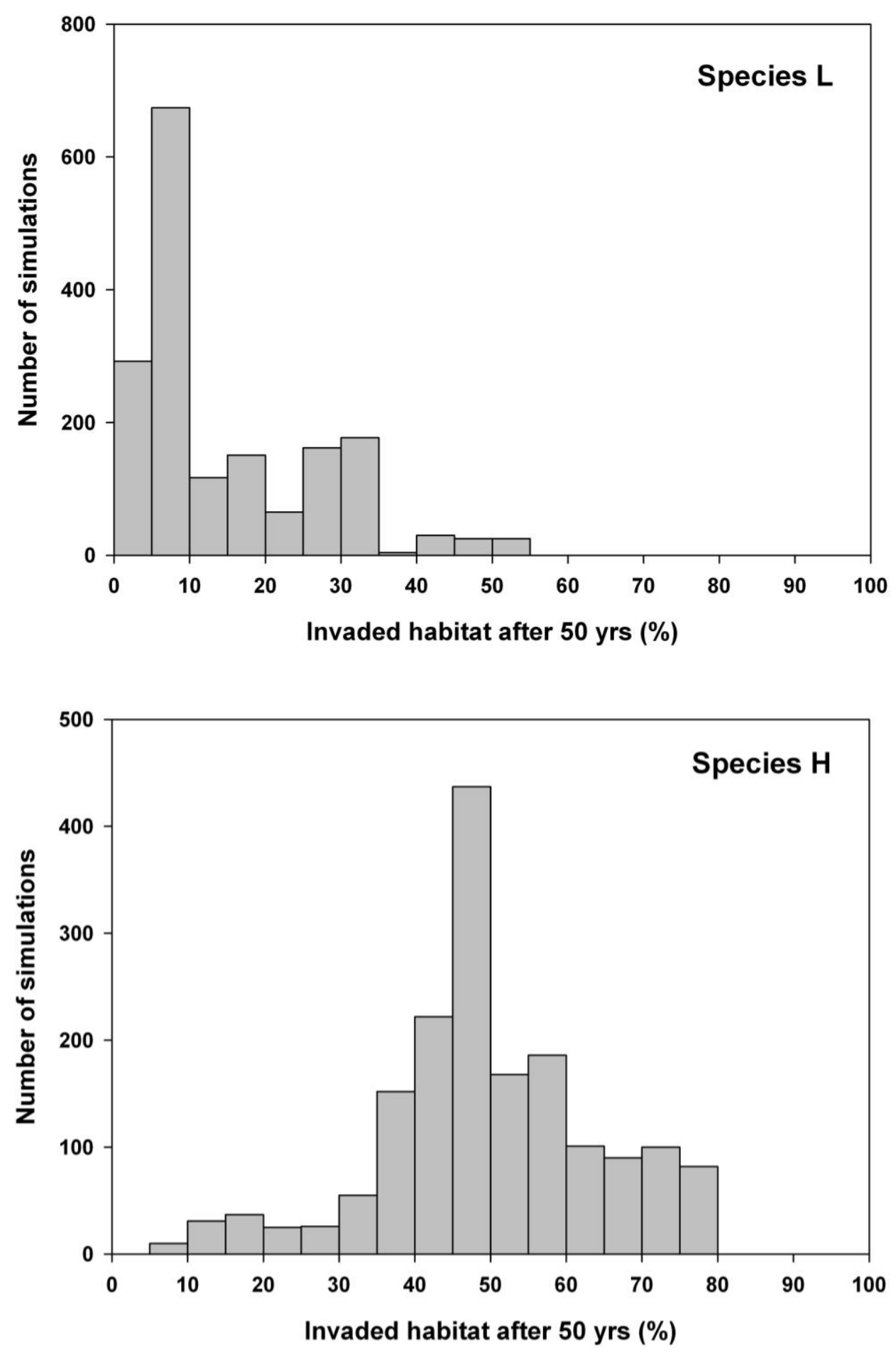

Figure 3 

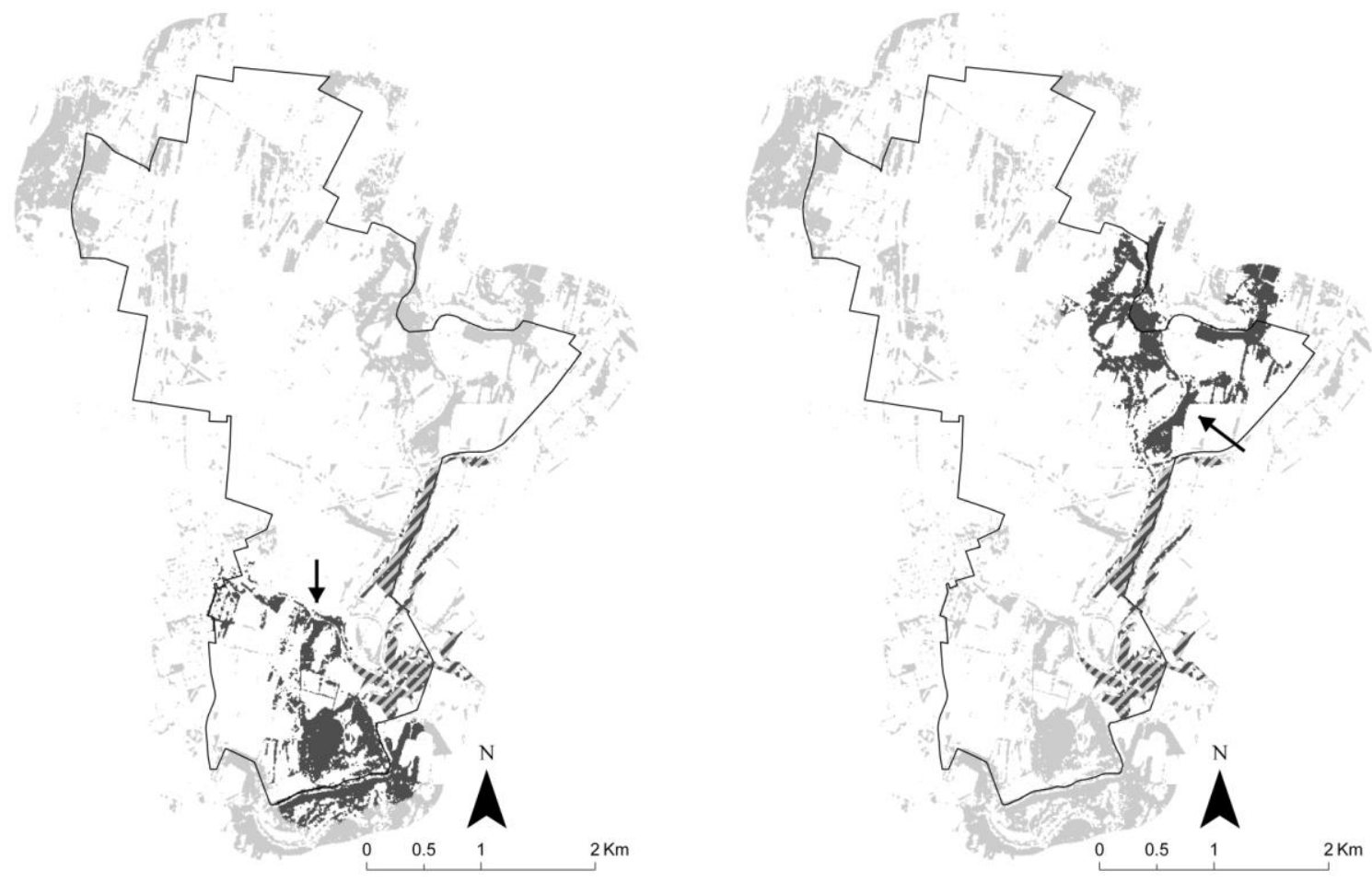

Figure 4 

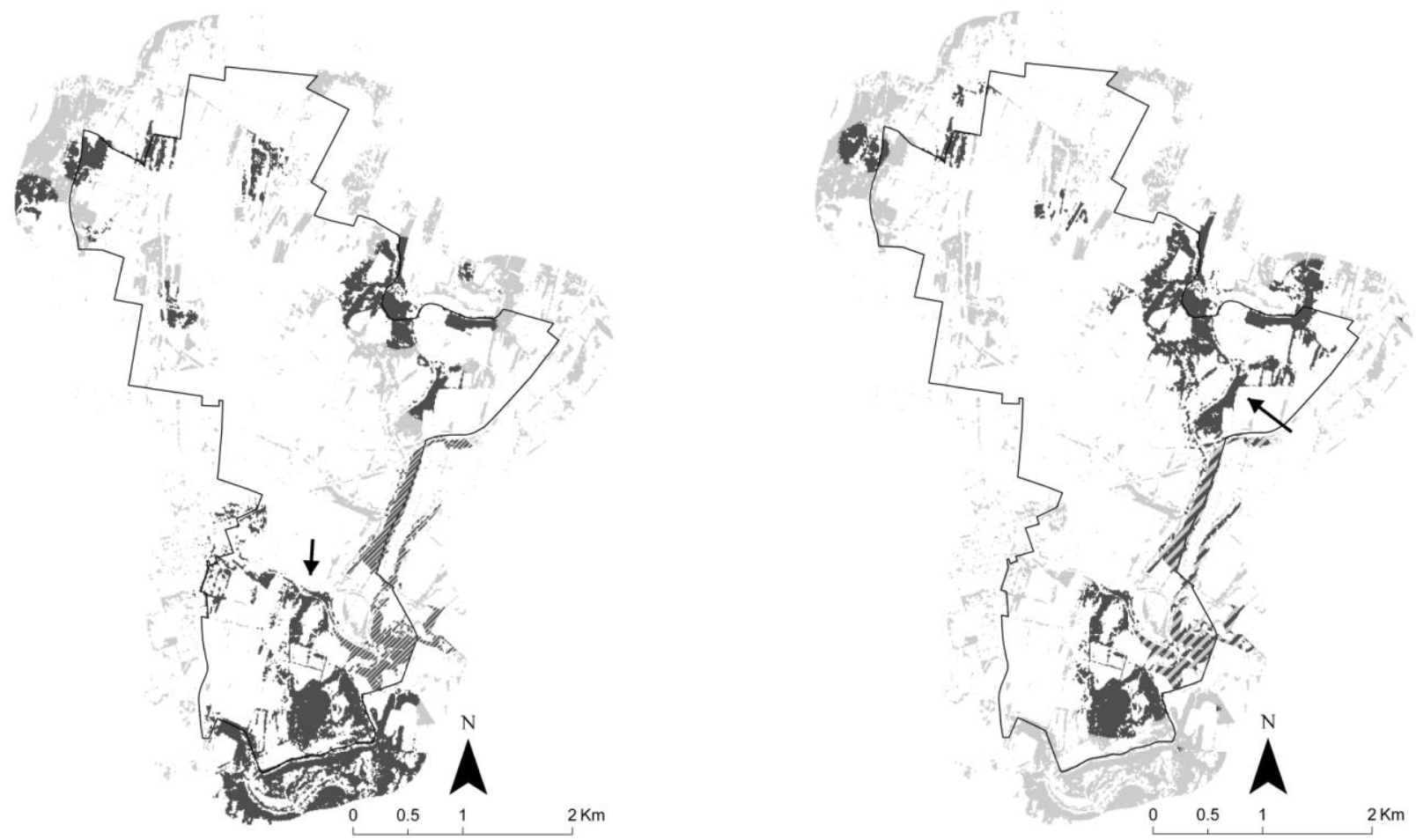

Figure 5 